\title{
Effectiveness of cognitive behavioral therapy on quality of life of patients with chronic low back pain
}

\author{
Maryam Didehdar Ardebil ${ }^{1 *}$ Atefeh Mohammad Nejad ${ }^{2}$
}

${ }^{1}$ Panjab University, Chandigarh, India

Email: didehdar@gmail.com

${ }^{2}$ Faculty of Medicine, Tehran University of Medical Sciences, Iran

*Corresponding author.

\section{Abstract}

Current evidence-based guidelines for treatments of chronic low back pain (CLBP) suggested including psychological aspects of back pain as well as physical treatments. Therefore, the current study targeted to examine the efficacy of cognitive behavioral therapy on quality of life of Iranian female patients with non-specific chronic low back pain (NSCLBP). During this pre-post experimental study fifty seven patients suffering from NSCLBP were randomly assigned either in CBT $(\mathrm{N}=28)$ or usual medical care groups ( $\mathrm{N}=29)$, respectively. The subjects drown from physiotherapy centers of Ardebil and assessed in three time frames; pre, post and one month after the intervention through standard questionnaire (SF12). Data obtained from the final samples $(\mathrm{N}=48)$ analyzed by analysis of covariance, clinical significance and effect size.The results of ANCOVA revealed that subjects in the CBT group indicated significant improvements in physical and mental components of quality of life in comparison with the usual care group. Effect sizes were .73 and .69 for mental and physical components of quality of life respectively. Clinical significance also showed that out of 24 patients in CBT group 14 patients recovered in time 1 and 15 patients in time 2 of evaluation respectively.CBT as an alternative therapy, including cognitive restructuring, relaxation and daily home works was beneficial in enhancement of physical and mental components of quality of life of female Iranian patients with NSCLBP.

Keywords: Behavioural therapy, Chronic LBP, Cognitive therapy, Psychological treatments

\section{Introduction}

Nonspecific or common chronic low back pain (NSCLBP) leads to major individual, clinical, social, and economic burdens in societies [1]. Generally high percentages of 
LBP patients are not satisfied with their usual medical care and they are involving in LBP related disabilities despite of receiving medicine. The bio-psychosocial model of pain developed to provide a conceptual rationale for including psychological intervention within pain management strategies to reduce patients pain in shorter time and in proper manner $[2-3,4]$.

It is generally accepted that chronic pain has a negative impact on quality of life and most of patients with CLBP experience low quality of life [5-6]. Quality of life impact wide range of physical and mental aspects of life such as mental health, emotional, social or physical roles etc. Several studies included quality of life as primary outcome variable in chronic pain related investigation and interventions. Though, the effectiveness of various psychological interventions such as cognitive behavior therapy (CBT), relaxation, yoga, hypnosis, biofeedback and mindfulness based therapies has been explored on quality of life of patients with chronic low back pain, still researchers suggest for further researches to explore the efficacy of different interventions across different cultures [7].

In the most available literature, CBT is one of the most promised psychotherapies method which revealed small to large effectiveness in different studies. The review of effectiveness of psychological interventions on chronic LBP provided support for the efficacy of psychological interventions on quality of life among LBP sufferers [8,9-10].

In a recent study Alda et al (2011 (assessed the effectiveness of CBT in comparison with pharmacological treatments and treatment as usual among patients with fibromyalgia. The result showed that CBT increased quality of life in comparison with pharmacological treatment and usual care, respectively [11]. A hallmark of this CBT approach is that individuals are taught new coping skills so that, over time, they become able to manage pain-related difficulties on their own [12]. Meanwhile, there are some studies with inconsistent result, for example, Dalton(2004) conducted a study on 131 patients receiving treatment who were randomly assigned in CBT or usual care groups. Patients in CBT group showed greater improvement at six-months post intervention with less average pain, better mental quality of life, and greater improvement in performance status; usual care patients showed little change [13]. Therefore, regarding the importance of level of quality of life on recovery of patients and inconsistency between previous researches in this area, We aimed to examine the efficacy of CBT protocol designed for pain management on physical and mental components of quality of life in compare of control group among Iranian females with NSCLBP. 


\section{Methods}

This study approved by the scientific committee of "Panjab University" and all patients who participated in the study signed consent form to participate in the study.This study used pre_ post experimental design to assess the efficacy of CBT on CLBP patients in three time frames (before-after- 4weeks after intervention). CBT program administered during 7 sessions (60-90 minutes) twice a week. The control group was not offered any type of intervention in the research project.

The initial samples of 109 were drawn from the archived files of all physiotherapy Centers' of Ardebil, (northern west of Iran), during September 2011-Janurary 2012. The samples included the patients' who were diagnosed as a NSCLBP patient by physicians and were under usual medical treatments. Out of initial sample $(\mathrm{N}=188)$ only 57 were given consent to participate in a research program. Participant were randomly assigned in small groups to receive CBT plus usual care $(\mathrm{N}=28)$ and only usual care $(\mathrm{N}=29)$. The randomization for assigning the patients in two groups was carried out according to the sealed envelope technique. Some patients dropped from the research program during and after the treatment because of the following reasons: incomplete questionnaire, session attendance less than 6 sessions, unavailability after intervention. The final sample of the study comprised of 48 Iranian females from physiotherapy centre's of Ardebil city.

Inclusion and exclusion criteria: $\quad$ Age $(30-45 ;$ Mean age $=40.3, \mathrm{SD}=7$. , Being under medical treatments (physiotherapy and medicine), Medical problem- history of NSCLBP\& persisting pain for at least6 months, Language- Persian, Gender- Female, Qualification- Educated at least up to high school, Consent and willingness to alternative and complementary therapies for pain management, Availability in the next three months, No history of Spine Surgery, No combination with other chronic disease, Who had psychotherapy in the last two years excluded

Intervention

First session: Education and information about intervention and back pain, listing a stress appraisal event in related worksheet

Second session: Identify pain related automatic thoughts

Third session: Evaluating identified automatic thoughts

Forth session: Challenging and replacing automatic thoughts with alternative thoughts

Fifth session: Relaxation exercise 
Sixth session: listing and engaging in new activities

Seventh session: Conclusion, feedbacks, post test [14].

\section{Outcome measure : Quality of Life (SF12)}

Quality of life assessed by the validated SF-12 Health Survey. It was developed as a shorter, quicker-to-complete alternative to the SF-36v2 Health Survey and measures the same eight health constructs. The constructs are: physical functioning; role physical; bodily pain; general health; vitality; social functioning; role emotional; and mental health. Items have five response choices (for example: all of the time, most of the time, some of the time, a little of the time, none of the time), apart from two questions for which there are three response choices (for the physical functioning domain). Four items are reverse scored. Summed raw scores in the eight domains are transformed to convert the lowest possible score to zero and the highest possible score to 100. Higher scores represent better health and well being. The standard form SF-12 uses a time frame of the past four weeks (15).

The Iranian version of SF12 surveyed in Iran indicated appropriate satisfactory internal consistency for both summary measures, that are the Physical Component Summary (PCS) and the Mental Component Summary (MCS); Cronbach's $\alpha$ for PCS-12 and MCS-12 was 0.73 and 0.72, respectively. The known - group comparison showed that the SF-12 discriminated well between men and women and those who differed in age and educational status $(\mathrm{P}<0.001)$. 2.5(17).

Analysis of Covariance (ANCOVA) is a statistical technique that allows us to look at the effect of an independent variable on a dependent variable, whilst patrolling out or removing the effect of another variable. This is done by reducing the error variance and adjusting the means on the dependent variable. For the present study, treatments (CBT and usual medical treatment) were independent variables and post test scores on quality of life questionnaire were dependent variables. For performing ANCOVA, the pre- test scores on these scales were used as covariates. The effect of interventions on quality of life was examined by reducing the error variance of pre-test scores and adjusting the means to post- test scores on the quality of life questionnaire. The Cohense $d$ effect size conducted also to see the size of the effect of intervention on patients who participated in the CBT group in comparison to the usual care group. The clinical significance method used to find out the number of patients who recovered out of total patients in experimental group [18]. 


\section{Results}

Descriptive statistic calculated in order to identify the percentage of NCLBP patients falling in high, moderate and low degree of the physical and mental quality of life out of a total population of NSCLBP patients (Female =48) in physiotherapy centres of Ardebil (Iran); $64.4 \%$ patients were low on physical quality of life, while $36.4 \%$ falling in the moderate physical quality of life category respectively and in term of mental quality of life $52.8 \%$ of the patients were low on mental quality of life, while $47.6 \%$ are falling in the moderate category respectively.

Quality of life Mental Component Scores(PCS)

The ANCOVA was calculated by first checking for homogeneity of regression of mental quality of life scores of treatment and control group. Hence, the assumption of homogeneity of regression was not violated. This indicates a liner relationship between the covariate(pre- test scores) and the independent variable (CBT).

Table 1: Tests of Between-Subjects Effects Dependent Variable

\begin{tabular}{|c|c|c|c|c|c|c|}
\hline בְ & Source & $\begin{array}{c}\text { Type III Sum of } \\
\text { Squares }\end{array}$ & Df & Mean Square & $F$ & Sig. \\
\hline \multirow{6}{*}{ 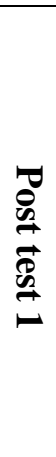 } & Corrected Model & $546.92^{\mathrm{a}}$ & 2 & 273.46 & 35.02 & .000 \\
\hline & Intercept & 161.76 & 1 & 161.76 & 20.71 & .000 \\
\hline & Group & 240.69 & 1 & 240.69 & 30.82 & .000 \\
\hline & Pre & 381.90 & 1 & 381.90 & 48.90 & .000 \\
\hline & Error & 351.39 & 45 & 7.80 & & \\
\hline & Total & 34131.00 & 48 & & & \\
\hline \multirow{6}{*}{ 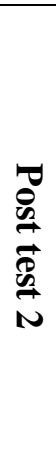 } & Corrected Model & $872.81^{\mathrm{a}}$ & 2 & 436.40 & 27.47 & .000 \\
\hline & Intercept & 406.38 & 1 & 406.38 & 25.58 & .000 \\
\hline & Group & 727.68 & 1 & 727.68 & 45.82 & .000 \\
\hline & Pre & 249.29 & 1 & 249.29 & 15.69 & .000 \\
\hline & Error & 714.66 & 45 & 15.88 & & \\
\hline & Total & 43065.00 & 48 & & & \\
\hline
\end{tabular}


The table shows that after adjusting for pre-test scores, there was a significant effect of the between subject factor group $\{\mathrm{F}(1,45)=30.82, \mathrm{p}<.001\}$ and $\{\mathrm{F}(1,45)=45.82, \mathrm{p}<$. $001\}$. Adjusted post test scores suggest that the intervention had an effect on increasing the mental quality of life scores of the NSCLBP patients who received the CBT as compared to those who were in the control group and did not receive any psychological therapy.

Quality of life, physical Component Scores (PCS)

The ANCOVA was calculated by first checking for homogeneity of regression of physical quality of life scores for experimental and control group. If the interaction between the covariate (pre-test scores) and the independent variable (CBT) is statistically significant then the data violates the assumption of homogeneity of regression slopes. In the present study, the interaction (group* pre-test) value $(\mathrm{F}=.319)$ for post testland $(\mathrm{F}=1.41)$ for post test 2 was not significant. Hence, the assumption of homogeneity of regression was not violated. This indicates a liner relationship between the covariate(pre- test scores) and the independent variable (CBT).

Table 2 Tests of Between-Subjects Effects Dependent Variable

\begin{tabular}{|c|c|c|c|c|c|c|}
\hline בְ & Source & Type III Sum of Squares & Df & Mean Square & $\mathrm{F}$ & Sig. \\
\hline \multirow{6}{*}{$\begin{array}{l}\overrightarrow{0} \\
\stackrel{2}{2} \\
\stackrel{\vec{\beta}}{2} \\
\stackrel{2}{\pi}\end{array}$} & Corrected Model & $255.91^{\mathrm{a}}$ & 2 & 127.95 & 31.31 & .000 \\
\hline & Intercept & 144.08 & 1 & 144.08 & 35.25 & .000 \\
\hline & Pre & 178.39 & 1 & 178.39 & 43.65 & .000 \\
\hline & Group & 42.05 & 1 & 42.05 & 10.29 & .002 \\
\hline & Error & 183.89 & 45 & 4.08 & & \\
\hline & Total & 24605.00 & 48 & & & \\
\hline \multirow{6}{*}{$\begin{array}{l}\vec{D} \\
\stackrel{D}{2} \\
\vec{\nabla} \\
\stackrel{2}{2} \\
\stackrel{N}{n}\end{array}$} & Corrected Model & $443.66 a$ & 2 & 221.83 & 29.20 & .000 \\
\hline & Intercept & 221.23 & 1 & 221.23 & 29.12 & .000 \\
\hline & Pre & 138.64 & 1 & 138.64 & 18.25 & .000 \\
\hline & Group & 233.69 & 1 & 233.69 & 30.76 & .000 \\
\hline & Error & 341.81 & 45 & 7.59 & & \\
\hline & Total & 27529.00 & 48 & & & \\
\hline
\end{tabular}


adjusting for pre-test scores, there was a significant effect of the between subject factor group $\{\mathrm{F}(1,45)=30.76, \mathrm{p}<.001\}$ and $\{\mathrm{F}(1,45)=10.29, \mathrm{p}<.001\}$. Adjusted post test scores suggest that the intervention had an effect on increasing the physical quality of life scores of the NSCLBP patients who received the CBT as compared to those who were in the control group and did not receive any psychological therapy.

Effect size: Patients who were exposed to CBT reported better physical and mental quality of life than $68 \%, 71 \%$ of patients in the control group who were not exposed to CBT intervention during time 1 evaluation.

Patients who were exposed to CBT reported better physical and mental quality of life than $71 \%, 70 \%$ of patients in the control group who were not exposed to the CBT intervention during time 2 evaluation

\section{Discussion}

This study surveyed the condition of physical and mental quality of life among patients with NSCLBP, descriptive analysis revealed a low level of physical and mental quality of life at baseline. The present study highlighted that around $62 \%$ of NSCLBP patients reported low to very low and the rest were moderate $(36.4 \%)$ on physical quality of life. Considering higher scores in quality of life indicates less pain, dysfunction or impairment this result implies that a high percentage of patients with NSCLBP experience high bodily pain and lower physical functioning. They are less able or unable to perform daily activities because of actual pain or fear of pain which gradually results in physical dysfunction.

In terms of mental components of quality of life (12.4\%), (40\%) and (47.6\%) of patients reported very low, low and moderate mental quality of life respectively. The results represented 52\% of NSCLBP patients are low to very low in mental quality of life and the rest is moderate. It means physical and social activity limitations in these patients resulted in a low level of mental health. In fact high level of fatigue and pain in low back pain patients is related to lower vitality and greater helplessness. Several studies confirm the negative impact of chronic pain on quality of life of patients with chronic pain. One Danish study reported that $45 \%$ of those with chronic non cancer pain rated their health as really good/good and 55\% rated their health as fair/bad/very In contrast, $88 \%$ of the control group who reported no chronic pain rated their health as really good/good bad [19-20]. 
The results of also showed CBT impacted both physical and mental components of quality of life significantly that sustained in one month after the intervention (posttest2) also. The patients reported higher physical function and lower pain after intervention. According to the "operant model" of CBT, the techniques used in CBT increase the social activities and patients received social reinforcement based on this model reinforced behaviors increases and ignored decreases [21]. Consequently, they reported less fatigue and high vitality. Obviously, these changes lead to the enhancement of general health among patients who participated in CBT sessions. Every technique delivered during the sessions of CBT has a notable role in the reduction of pain and enhancement of quality of life. In first two sessions the patients find out about the interference of maladaptive pain related thoughts in their daily life through identifying pain related automatic thoughts. In the next sessions, through cognitive restructuring, they generated the evidence that helped them in supporting or rejecting a particular pain related belief (e.g. I can't climb stairs). After using a rating system of each pain related belief, the patients discovered that their beliefs about pain were faulty and needed to be changed. Subsequently patients looked for positive alternative thoughts, which could be replaced with negative ones. Thereby they developed new positive ways of thinking about pain which did not only reduce pain but also helped them to promote their quality of life.

Relaxation also helped them in the improvement of quality of life through reducing their physical and mental anxiety. Lower stress and anxiety is related to a higher mental and functional ability. In addition reviewing the completed daily homework's in a group help them to discover the common daily maladaptive coping styles which are the cause of functional and social limitations among patients with chronic LBP. This awareness encouraged them to attend to their daily life demands instead of getting overwhelmed with pain related thoughts.

A promise of CBT approach is that individuals learn new coping skills so that, over time, they become capable of managing pain-related difficulties on their own. The findings from numerous studies of persons with chronic pain indicate that individuals' beliefs about pain are associated with various indices of functioning. Researches also supports the hypothesis that changes in patients' beliefs about pain are associated with changes in functioning, consistent with the hypothesis that patient's cognitions play a key role in adjustment to chronic pain and boosting the quality of life level. Following researches supports the contention that cognitive behavioral programs lead to enhancement of quality of life in patients with chronic. 
Some of the previous research supports the hypothesis that changes in patients' beliefs about pain are associated with changes in functioning, consistent with the hypothesis that patient's cognitions play a key role in adjustment to chronic pain and boosting the quality of life level [22]. Following researches supports the contention that cognitive behavioral programs lead to enhancement of quality of life in patients with chronic pain.

Darren et al,. (2008) evaluated a study in 360 of patients referred to a hospital pain clinic that completed a CBT programmer. Program applied cognitive behavioral (CB) principles in all treatment components including physical upgrading, the cessation of pain-related medication and other aides, education and training in the self-management of chronic pain. The study demonstrates improvements across physical and psychological measures post-CBT [23]. Jonsbu et al,.(2011) carried out a study on 40 patients with non-cardiac chest pain to compare a three-session CBT intervention with normal care for patients with non-cardiac chest pain. Result manifested significantly larger improvements in the treatment group regarding fear of bodily sensations, avoidance of physical activity, depression and some domains of health related quality of life at the end of treatment, and at three- and 12-month follow-up [11].

Lamb et al., (2012) undertook an extended follow-up of original randomized, controlled trial of a group CBT and best practice advice in primary care, on 56 participants for six weeks in comparison to best practice advice alone. The time of extended follow-up ranged between 20 and 50 months (mean 34 months). After 12 months, the improvements in pain and disability observed with CBT were sustained. For disability measures, the treatment difference in favour of CBT persisted. The results suggest that the effects of a group CBT are maintained up to an average of 34 months [24]. Andersson et al,.(2012) did a study of 21 persons (mean age $=72.0$ years) who were randomly allocated to either a waitlist condition or CBT. Few statistically significant effects were found on measures of pain, mood, anxiety, and quality of life; however, a significant treatment effect with a between group effect size of $d=1.0$ was observed with respect to perceived ability to function despite the discomfort of pain [25].

Although this study is limited by its small sample size and short time for follow-up, but our results have important implications for the design of clinical trials in CBT research, including the importance of piloting the control group before running a large clinical trial. Future studies with homogeneous chronic pain conditions are needed to clarify to which techniques most and least effective and what are underlying mechanisms account for variation in outcome. 


\section{References}

1. Koes BW, van Tulder MW, Thomas S. Diagnosis and treatment of low back pain. BMJ. 2006 17;332(7555):1430-4.

2. Waddell G, (1998). The Back Pain Revolution. London, England, Churchill Livingstone.

3. I Innes S. Psychosocial factors and their role in chronic pain: A brief review of development and current status. Chiropr Osteopat 2005, 13:6

4. Glombiewski JA, Hartwich-Tersek J, Rief W. Two Psychological Interventions Are Effective in Severely Disabled, Chronic Back Pain Patients: A Randomised Controlled Trial. INT J BEHAV MED. 2010, 17(2): 97-107

5. Nakao M, Shinozaki Y, Nolido N, Ahern DK, Barsky AJ. Group cognitive behavioural interventions for low back pain in primary care: Pain 2012; 153: 494-501

6. Schlenk EA, Erlen JA, Dunbar ,Jacob J, McDowell J, Engberg S, Sereika SM, et al: Health-related quality of life in chronic disorders: a comparison across studies using the MOS SF-36. Quality of Life Research. 1998, 7(1):57-65.

7. Veehof MM, Oskam MJ, Schreurs KM, Bohlmeijer ET. Acceptance-based interventions for the treatment of chronic pain: a systematic review and meta-analysis: Pain 2011, 152(3):533-42.

8. Henschke N, Ostelo RW, van Tulder MW, Vlaeyen JW, Morley S, Assendelft WJ, Main CJ. Behavioural treatment for chronic low-back pain. Cochrane Database Syst Rev. 2010;(7).

9. Smeets RJ, Vlaeyen JW, Hidding A, et al. Chronic low back pain: physical training, graded activity with problem solving training, or both? The one-year posttreatment results of a randomized controlled trial. Pain. 2008,134:263-276.

10. Wetherell JL, Afari N, Rutledge T, Sorrell JT, Stoddard JA, Petkus AJ, Solomon BC, Lehman DH, Liu L, Lang AJ, Atkinson JH (2011). A randomized, controlled trial of acceptance and commitment therapy and cognitive-behavioral therapy for chronic pain. Pain, 152(9):2098-107.

11. Jonsbua E, Dammenc T, Morkenb G, Moumc T \& Martinsend EW. Short-term cognitive behavioral therapy for non-cardiac chest pain and benign palpitations: A randomized controlled trial. J Psychosom Res, 2011, 70 (2), 117-123. 
12. Alda M, Luciano JV, García-Campayo J. Effectiveness of cognitive behaviour therapy for the treatment of catastrophisation in patients with fibromyalgia: a randomised controlled trial. Arthritis Research \& Therapy, 2011, 13(5); 173.

13. Osborne TL, Raichle KA, Jensen MP.Psychologic Interventions for Chronic Pain. Phys Med Rehabil Clin N Am, 2006, 17: 415-433 .

14. Dalton JA, Keefe FJ, Carlson J, Youngblood R. Tailoring cognitive-behavioral treatment for cancer pain. Pain Manag Nurs. 2004, 5(1):3-18.

15. Beverly E. Thorn. Cognitive therapy for chronic pain: a step-by-step guide. USA, 2004 The Guilford Press

16. Ware JE, Jr., Kosinski M, Turner-Bowker DM, Gandek B. How to Score Version 2 of the SF-12® Health Survey (With a Supplement Documenting Version 1) Lincoln, RI: Quality Metric Incorporated; 2002

17. Montazeri A, Vahdaninia M, Mousavi SJ \& Omidvari S. The Iranian version of 12-item Short Form Health Survey (SF-12): a population-based validation study from Tehran, Iran. Health Qual Life Outcomes 2011, 9:12

18. Jacobson N, Truax P. Clinical significance: A statistical approach to defining meaningful change in psychotherapy research. J Con Clin Psy, 1991, 59(1), 12-19.

19. Eriksen J, Sjøgren P, Bruera E, Ekholm O, and RasmussenNK. Critical issues on opioids in chronic non-cancer pain: an epidemiological study. Pain, 2006, 125(12): 172179.

20. Sjøgren P, Ekholm O, Peuckmann V, Grønbaek M. Epidemiology of chronic pain in Denmark: an update.Eur J Pain. 2009,13(3):287-92.

21.Coupland M . CBT for pain management. IAIABC Journal. 2009, 46 ( 2), 77-78.

22. Jensen IB, Bergstro“m G, Ljungquist T, Lennart Bodin. A 3-year follow-up of a multidisciplinary rehabilitation programme for back and neck pain. Pain, 115 (2005) 273-283

23. .Darren C. White, Rowena Beecham, Kathryn Kirkwood. The Vocational Continuum: How to Make Sense of Vocational Outcomes After Group Cognitive Behavioural Therapy for Chronic Pain Sufferers. J Occup Rehabil, 2011, 18 (3): 307317. 
24. Lamba SE, MistryaD, Lalla R, Hansena Z, Evansa D, Withersa EJ, Underwooda MR. Group cognitive behavioural interventions for low back pain in primary care: Extended follow-up of the Back Skills Training. Pain, 153(2): 494, 502.

25. Anderson, Renee E. E., Spence, Susan H., Donovan, Caroline L., March, Sonja, Prosser, Samantha and Kenardy, Justin. A working alliance in online cognitive behaviour therapy for anxiety disorders in youth: Comparison with clinic delivery and its role in predicting outcome. J Med Internet Res, 2012, 143: 82-97.

\section{List of Abbreviation}

$\mathrm{LBP}=$ low back pain

$\mathrm{CBT}=$ cognitive behavior therapy

$\mathrm{NSCLBP}=$ non-specific chronic low back pain 